\title{
Effect of Parallel Magnetic Field on Superconductivity of Ultrathin Metal Films Grown on a Cleaved GaAs Surface
}

\author{
Takayuki Sekihara, Takahiro Miyake, Ryuichi Masutomi, and Tohru Okamoto
}

Department of Physics, University of Tokyo, 7-3-1 Hongo, Bunkyo-ku, Tokyo 113-0033, Japan

\begin{abstract}
The parallel-magnetic-field $H_{\|}$dependence of the superconducting transition temperature $T_{c}$ is studied for ultrathin films of In, Bi, and $\mathrm{Al}$ grown on $\mathrm{GaAs}(110)$. In the case of In films in the monolayer regime, $T_{c}$ exhibits a quadratic-like $H_{\|}$dependence, which is one order of magnitude stronger than that previously observed in monolayer $\mathrm{Pb}$ films by the present authors [Phys. Rev. Lett. 111, 057005 (2013)]. The results are well reproduced by a model developed for an inhomogeneous two-dimensional superconducting state in the presence of a moderate Rashba spin-orbit interaction. The Rashba spin splitting is estimated to be $0.04 \mathrm{eV}$, which is much smaller than that expected for monolayer $\mathrm{Pb}$ films. In a few-monolayer Bi film, the suppression of $T_{c}$ with increasing $H_{\|}$is comparable to that in monolayer $\mathrm{Pb}$ films. On the other hand, much stronger suppression, which is attributed to the Pauli paramagnetic effect, was observed for the Al film.
\end{abstract}

\section{Introduction}

Recently it has been demonstrated that superconductivity can occur even in one-atomic-layer metal films. ${ }^{1,2)}$ In an ultrathin film grown on a substrate, the asymmetry of the confining potential is expected to cause the Rashba spin-orbit interaction. ${ }^{3,4)}$ It was shown in Ref. 5 that the superconducting critical magnetic field in the parallel direction, where the orbital effect is absent, can be very high for a two-dimensional system with a large Rashba spin splitting $\Delta_{R}$ because of the formation of an inhomogeneous superconducting state similar to the Fulde-Ferrell-Larkin-Ovchinnikov state. ${ }^{6,7)}$ This FuldeFerrell-Larkin-Ovchinnikov-like state is easily destroyed by disorder. ${ }^{8,9)}$ Instead, a different inhomogeneous superconducting state appears, which is called the long-wavelength helical state. ${ }^{8,9)}$ Here the Cooper pairs have a very small but nonzero momentum $\mathbf{q}$ and the order parameter varies as $\exp (i \mathbf{q} \cdot \mathbf{r})$. Very recently, the present authors studied the effect of a parallel magnetic field $H_{\|}$on the superconductivity of monolayer $\mathrm{Pb}$ films on $\mathrm{GaAs}(110) .{ }^{10)}$ Superconductivity was found to occur even for $H_{\|}=14 \mathrm{~T}$, which is much higher than the Pauli paramagnetic limiting field $H_{P}{ }^{11,12)}$ The observed weak $H_{\|}$dependence of the superconducting transition temperature $T_{c}$ is well reproduced by

$$
T_{c}=T_{c 0}-\frac{\pi \tau}{2 k_{B} \hbar}\left(\mu_{B} H_{\|}\right)^{2},
$$

where $T_{c 0}$ is the zero-field value of $T_{c}, \tau$ is the elastic scattering time, and $\mu_{B}$ is the Bohr magneton. This expression is derived from the theory of Refs. 8 and 9 developed for the case $\Delta_{R} \gg \hbar \tau^{-1}$.

The strength of the spin-orbit interaction strongly depends on the atomic number $Z$. In order to confirm the origin of the robustness of superconductivity against $H_{\|}$in $\mathrm{Pb}$ films $(Z=83)$, it is important to study ultrathin superconducting films of other materials. In this work, we extend our studies to ultrathin films of In $(Z=49), \mathrm{Bi}(Z=83)$, and $\mathrm{Al}$ $(Z=13)$. In the case of In films, superconductivity was observed in the monolayer regime. The $H_{\|}$dependence of $T_{c}$ is one order of magnitude stronger than that in the $\mathrm{Pb}$ films and does not quantitatively agree with Eq. (1). Since $\Delta_{R}$ is expected to be small in the In films, we also extend the analysis of Refs. 8 and 9 to the case where $\Delta_{R}$ is comparable to or smaller than $\hbar \tau^{-1}$. The experimental results are well reproduced by the calculation with $\Delta_{R} \approx 0.04 \mathrm{eV}$, which is much smaller than that expected for the $\mathrm{Pb}$ films. For $\mathrm{Bi}$ and $\mathrm{Al}$, superconducting monolayer films were not obtained. We measured the $H_{\|}$dependence of $T_{c}$ for the smallest thickness for which superconductivity was observed. In the Bi film, it is as weak as that observed in the monolayer $\mathrm{Pb}$ films. In the $\mathrm{Al}$ film, on the other hand, it is strong and can be explained in terms of the Pauli paramagnetic effect.

\section{Experimental Methods}

The sample preparation methods and experimental setup are similar to those previously described. ${ }^{10)}$ We used a nondoped insulating GaAs single-crystal substrate so as not to create conduction channels in the substrate. Current and voltage electrodes were prepared at room temperature by the deposition of gold films onto noncleaved surfaces. The cleavage of GaAs, the deposition of an ultrathin film by quench condensation, and resistance measurements were performed at low temperatures in an ultrahigh-vacuum chamber immersed in liquid He. The amount deposited was measured with a quartz crystal microbalance and determined with an accuracy of about 5\%. The four-probe resistance of the ultrathin film on a cleaved $\operatorname{GaAs}(110)$ surface $\left(4 \times 0.35 \mathrm{~mm}^{2}\right)$ was measured using the standard lock-in technique at $13.1 \mathrm{~Hz}$. The magnetic-field direction with respect to the surface normal was precisely controlled using a rotatory stage on which the sample was mounted, together with a Hall generator, a $\mathrm{RuO}_{2}$ resistance thermometer, and a heater. The sample stage can be cooled to $0.5 \mathrm{~K}$ via a silver foil linked to a pumped ${ }^{3} \mathrm{He}$ refrigerator. All the data were taken when the temperature of the sample stage was kept constant so as to ensure thermal equilibrium between the sample and the thermometer. The magnetoresistance effect of the $\mathrm{RuO}_{2}$ resistance thermometer was systematically calibrated against the vapor pressure of the liquid ${ }^{3} \mathrm{He}$ or ${ }^{4} \mathrm{He}$ for various temperatures. After the correction, $T_{c}$ can be determined with a relative accuracy of better than $0.2 \%{ }^{13)}$ 


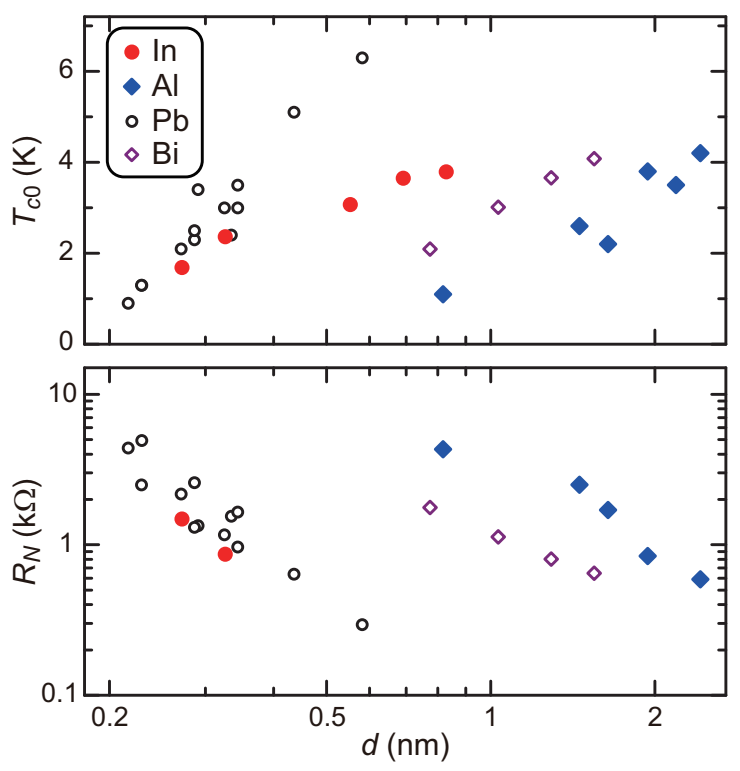

Fig. 1. (Color online) Zero-field superconducting transition temperature and normal-state sheet resistance as a function of the nominal thickness of quench-condensed films on GaAs(110). Data for $\mathrm{Pb}$ and Bi are from Ref. 10.

\section{Results and Discussion}

\subsection{Thickness dependence}

Figure 1 shows the zero-field superconducting transition temperature $T_{c 0}$ and the normal-state sheet resistance $R_{N}$ as a function of the nominal thickness $d$ of quench-condensed films of In, $\mathrm{Al}, \mathrm{Pb}$, and $\mathrm{Bi}$. We defined the transition temperature as the temperature at which the sheet resistance $R_{s q}$ reaches $0.5 R_{N}$. Compared with the results on a glazed alumina substrate coated with amorphous Ge reported in Ref. 14, $R_{N}$ is lower and $T_{c 0}$ is higher for $\mathrm{Pb}$ and $\mathrm{Bi}^{10)} \mathrm{We}$ attribute this to the atomically flat surface of the cleaved GaAs substrate. Films of In and $\mathrm{Pb}$ show superconductivity even in the monolayer regime $(d \lesssim 0.3 \mathrm{~nm}$ ). For $\mathrm{Al}$ and $\mathrm{Bi}$, on the other hand, we could not attain a superconducting monolayer film. The morphology of the Al film will be discussed in Sect. 3.4.

\subsection{In films}

Figure 2 shows the temperature dependence of the sheet resistance $R_{s q}$ of In films with atomic areal densities $n=10.4$ and $12.5 \mathrm{~nm}^{-2}$. The corresponding values of $d$ are 0.27 and $0.32 \mathrm{~nm}$, respectively, with a bulk density of $38.3 \mathrm{~nm}^{-3}$. Since they are close to the cube root of the volume per atom in bulk indium $(0.30 \mathrm{~nm})$, the films are considered to be almost monolayer. In contrast to epitaxially grown films, ${ }^{1,2)}$ the quenchcondensed films are expected to be amorphous or highly disordered. ${ }^{14)}$ The normal-state sheet resistances $R_{N}$ in our In films are higher than $410 \Omega$, the value for the monolayer In film grown epitaxially on $\mathrm{Si}(111)$ by Uchihashi et al. ${ }^{2)}$ As in the case of the $\mathrm{Pb}$ films, ${ }^{10}$ ) superconductivity is observed even for $H_{\|}$higher than the Pauli paramagnetic limiting field $H_{P}(\mathrm{~T})=1.86 T_{c 0}(\mathrm{~K}) .{ }^{11,12)}$ However, the effect of $H_{\|}$on $T_{c}$ is much stronger than that in the $\mathrm{Pb}$ films.

In the presence of the perpendicular component $H_{\perp}$ of the magnetic field, the superconducting state is easily destroyed because of the orbital effect. Figure 3 shows the $H_{\perp}$ dependence of $R_{s q}$ for the In film with $n=12.5 \mathrm{~nm}^{-2}$ at $T=0.83 \mathrm{~K}$.

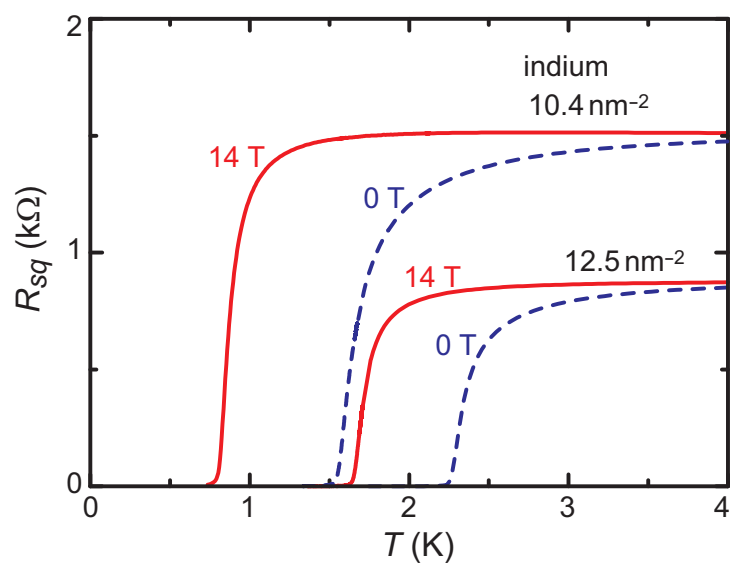

Fig. 2. (Color online) $T$ dependence of the sheet resistance of In films for two atomic areal densities. Dashed (blue) curves are obtained at zero magnetic field. Solid (red) curves are obtained in a parallel magnetic field of $14 \mathrm{~T}$.

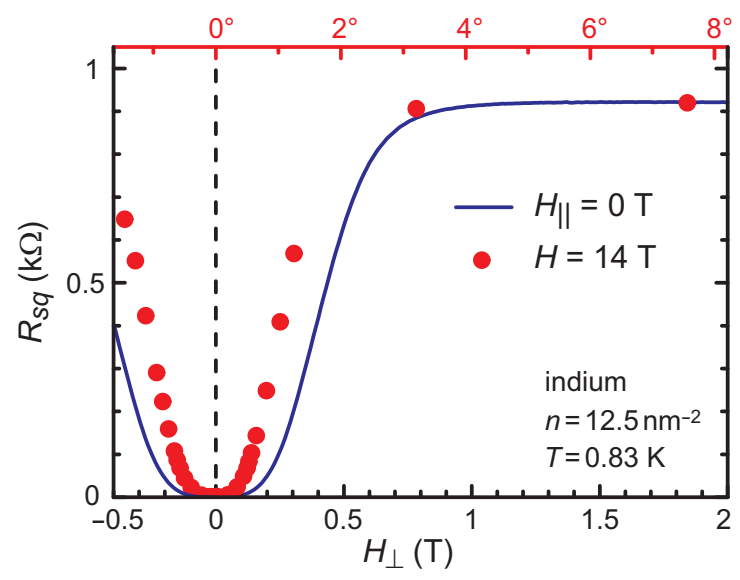

Fig. 3. (Color online) $H_{\perp}$ dependence of the sheet resistance of the In film with $n=12.5 \mathrm{~nm}^{-2}$ at $T=0.83 \mathrm{~K}$. The solid (blue) curve is obtained for the perpendicular-magnetic-field direction $\left(H_{\|}=0\right)$. Filled (red) circles are obtained by changing the magnetic-field angle (upper axis) at a fixed strength of $14 \mathrm{~T}$.

Data obtained for $H_{\|}=0$ (solid curve) and $H_{\|} \approx H=14 \mathrm{~T}$ (filled circles) are compared. While the $H_{\perp}$ dependence of $R_{s q}$ was found to be almost independent of the presence of $H_{\|}$in the case of the $\mathrm{Pb}$ films, ${ }^{10)}$ the suppression of superconductivity by $H_{\|}$is apparent for the In films. Since $H_{\|}$does not change the Lorentz force acting on vortices, the difference in $R_{s q}$ for low $H_{\perp}$ is attributed to that in the pinning force, which should be related to the superconducting gap.

In Fig. 4, the $H_{\|}$dependence of $\Delta T_{c} \equiv T_{c}-T_{c 0}$ is shown for the In films. Data for the $\mathrm{Pb}$ films ${ }^{10)}$ are also plotted for comparison. As in the $\mathrm{Pb}$ films, $T_{c}$ exhibits a quadratic-like $H_{\|}$dependence, which is at least qualitatively consistent with Eq. (1). However, the suppression of $T_{c}$ is one order of magnitude stronger than that in the $\mathrm{Pb}$ films. If Eq. (1) is applied to the experimental data, $\tau$ is determined to be 43 and 35 fs for $n=10.4$ and $12.5 \mathrm{~nm}^{-2}$, respectively. These values are significantly larger than $\tau=2.8$ and $4.0 \mathrm{fs}$, roughly estimated from $R_{N} \cdot{ }^{15)}$ We attribute this discrepancy to the fact that Eq. (1) is derived for $\Delta_{R} \gg \hbar \tau^{-1}$. Since the Rashba spin splitting originates both from the asymmetry of the confining potential and 


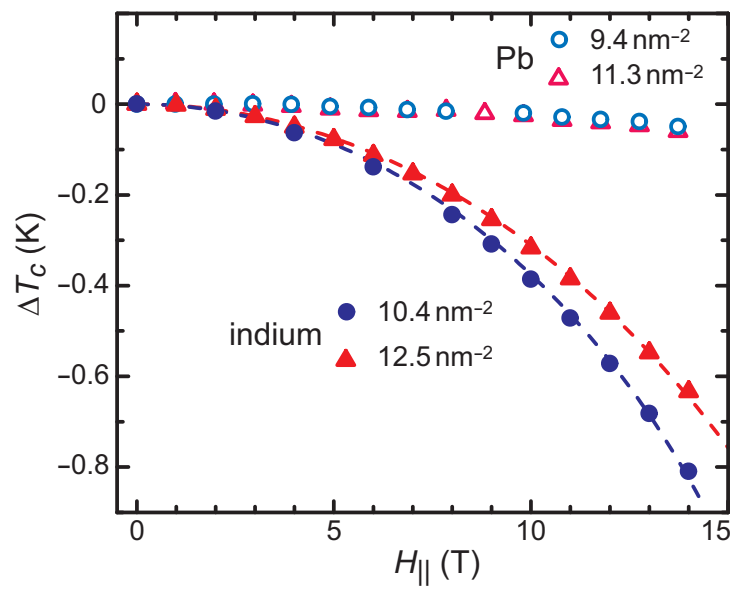

Fig. 4. (Color online) $H_{\|}$dependence of $\Delta T_{c} \equiv T_{c}-T_{c 0}$. Solid symbols represent data for the In films. Open symbols are data from Ref. 10 for the $\mathrm{Pb}$ films. The dashed lines represent the best fits to Eq. (6).

atomic spin-orbit coupling, $\Delta_{R}$ is expected to be small for elements with small atomic numbers $Z$. In the following, we consider the case where $\Delta_{R}$ is smaller than $\hbar \tau^{-1}$.

The stability of the long-wavelength helical state ${ }^{8,9)}$ can be interpreted as being due to mixing of the spin-up state and spin-down state, which weakens the pair-breaking effect of $H_{\|} \cdot{ }^{16)}$ For a qualitative understanding, it is helpful to use the analogy with the theory developed for the case where the spin mixing is caused by the spin-orbit scattering of impurities. ${ }^{17,18)}$ For a superconductor with strong spin-orbit scattering, $T_{c}$ is given by

$$
\ln \left(\frac{T_{c}}{T_{c 0}}\right)+\psi\left(\frac{1}{2}+\frac{3 \tau_{\mathrm{SO}}\left(\mu_{B} H_{\|}\right)^{2}}{4 \pi \hbar k_{B} T_{c}}\right)-\psi\left(\frac{1}{2}\right)=0,
$$

where $\tau_{\mathrm{SO}}$ is the spin-orbit scattering time and $\psi(x)$ is the digamma function. For $T_{c} \approx T_{c 0}$, the $H_{\|}$dependence of $T_{c}$ is obtained as

$$
T_{c}=T_{c 0}-\frac{3 \pi \tau_{\mathrm{SO}}}{8 k_{B} \hbar}\left(\mu_{B} H_{\|}\right)^{2} .
$$

In the present case, we consider nonmagnetic elastic scattering by defects, which conserves the spin of the conduction electrons. ${ }^{10)}$ However, the subsequent spin precession due to the Rashba effect can change the spin direction. If the precession frequency $\Omega_{R}=\hbar^{-1} \Delta_{R}$ is high enough $\left(\Omega_{R} \tau \gg 1\right)$, the effective spin relaxation time $\tau_{s}^{*}$ is expected to be comparable to $\tau$. Actually, Eq. (3) is similar to Eq. (1) when $\tau_{\text {SO }}$ is replaced by $\tau_{s}^{*} \sim \tau$, while the origin of the spin relaxation is different. For $\Omega_{R} \tau \ll 1$, on the other hand, the electron spin rotates by a small angle $\Omega_{R} \tau$ between successive scattering events and follows a random walk. This is the D'yakonov-Perel mechanism ${ }^{19,20)}$ and $\tau_{s}^{*} \sim\left(\Omega_{R}^{2} \tau\right)^{-1} \gg \tau$ is obtained. In this case, by analogy with Eq. (3), the coefficient of the quadratic $H_{\|}$ dependence of $T_{c}$ can be much larger than that in Eq. (1).

For a quantitative description, we here extend the analysis of Refs. 8 and 9 to the case $k_{B} T_{c 0} \ll \Delta_{R} \lesssim \hbar \tau^{-1} \ll \epsilon_{F}$, where $\epsilon_{F}$ is the Fermi energy. We take into account Cooper blocks ${ }^{9)}$ where the chiralities of the two electrons are different, while their contributions are negligible for $\Delta_{R} \gg \hbar \tau^{-1}$. Equation (13) of Ref. 8, which represents the Cooper kernel ${ }^{8,9)}$ as a function of the Matsubara frequency $\omega$ and is equivalent to
Eq. (94) of Ref. 9, is replaced by

$$
K(\omega)=4 \tau \frac{I_{s}^{0}\left[1-I_{s}^{2}-J\right]+\left(I_{a}^{1}\right)^{2}}{\left(1-I_{s}^{0}\right)\left[1-I_{s}^{2}-J\right]-\left(I_{a}^{1}\right)^{2}},
$$

where we introduce

$$
J=\frac{\hbar^{2}}{4 \tau} \frac{\bar{\omega}}{\hbar^{2} \bar{\omega}^{2}+\left(\alpha p_{F}\right)^{2}}=\frac{1}{\tau} \frac{\bar{\omega}}{4 \bar{\omega}^{2}+\Omega_{R}^{2}},
$$

with $\bar{\omega}=\omega+1 / 2 \tau$. After some algebra, we obtain

$$
\frac{T}{T_{c 0}}=\exp \left[-\frac{2 \pi k_{B} T}{\hbar} \sum_{\omega>0}^{\infty} \frac{1}{\omega} \frac{2 \tau_{s}^{*} h_{\|}^{2}}{\omega^{2} \tau^{\prime}+\omega+2 \tau_{s}^{*} h_{\|}^{2}}\right],
$$

with $h_{\|}=\mu_{B} H_{\|} / \hbar, \tau^{\prime}=4 / \Omega_{R}^{2} \tau$, and

$$
\tau_{s}^{*}=\left(1+\frac{1}{2 \Omega_{R}^{2} \tau^{2}}\right) \tau
$$

By solving Eq. (6) for $T, T_{c}$ is numerically determined as a function of $H_{\|}$. For small magnetic fields $\left(\left|\Delta T_{c}\right| \ll T_{c 0}\right)$, it is given by

$$
T_{c}=T_{c 0}-\frac{\pi \tau_{s}^{*} f(a)}{2 k_{B} \hbar}\left(\mu_{B} H_{\|}\right)^{2},
$$

where

$$
f(a)=1-\frac{2 a}{\pi^{2}}\left[\psi\left(\frac{1}{2}+\frac{1}{a}\right)-\psi\left(\frac{1}{2}\right)\right]
$$

with

$$
a=\frac{8 \pi \hbar k_{B} T_{c 0}}{\tau \Delta_{R}^{2}}
$$

For $\Delta_{R} \gg \hbar \tau^{-1}\left(\Omega_{R} \tau \gg 1\right)$, we have $\tau_{s}^{*} \approx \tau, a \approx 0, f(a) \approx 1$, and then Eq. (8) agrees with Eq. (1). This condition is expected to be satisfied for the $\mathrm{Pb}$ films since the values of $\tau$ estimated from $R_{N}$ are in good agreement with those obtained from the $H_{\|}$dependence of $T_{c}$ using Eq. (1). ${ }^{10)}$ In the opposite limit $\Delta_{R} \ll \hbar \tau^{-1}$, we obtain $\tau_{s}^{*} \approx\left(2 \Omega_{R}^{2} \tau\right)^{-1}$, which is consistent with the D'yakonov-Perel model. For $\Delta_{R} \ll \hbar \tau^{-1}$ and $\Delta_{R}^{2} \lesssim\left(k_{B} T_{c 0}\right)\left(\hbar \tau^{-1}\right)$, the reduction of $f(a)$, which arises from the $\omega^{2} \tau^{\prime}$ term in Eq. (6), should be taken into account.

The dashed lines in Fig. 4 are the best fits to Eq. (6). Here we use $\tau=2.8$ and $4.0 \mathrm{fs,}$, which are estimated from $R_{N}$. Our calculation well reproduces the experimental results. The obtained values of $\Delta_{R}$ are 39 and $35 \mathrm{meV}$ for the In films with $n=10.4$ and $12.5 \mathrm{~nm}^{-2}$, respectively. ${ }^{21)}$ They are one order of magnitude smaller than those measured for monolayer Bi (Refs. 22-24) and $\mathrm{Pb}$ (Ref. 25) films grown on $\mathrm{Si}(111)$ or Ge(111) by angle-resolved photoelectron spectroscopy. For our $\mathrm{Pb}$ films on $\operatorname{GaAs}(110),{ }^{10)} \Delta_{R}$ is expected to be larger or at least comparable to $\hbar \tau^{-1} \sim 0.2 \mathrm{eV}$ since the observed $H_{\|}$ dependence of $T_{c}$ agrees with Eq. (1), which is derived for $\Delta_{R} \gg \hbar \tau^{-1}$. It has been shown by a tight-binding calculation that the Rashba parameter is proportional to the product of the gradient of the surface potential and the magnitude of the atomic spin-orbit coupling. ${ }^{26)}$ Since the latter scales as $Z^{4}$, it seems likely that $\Delta_{R}$ varies by one order of magnitude from In $(Z=49)$ to $\mathrm{Pb}(Z=82)$ or $\mathrm{Bi}(Z=83)$.

\subsection{Bi films}

Figure 5 shows the $H_{\|}$dependence of $T_{c}$ for the Bi film with $d=0.77 \mathrm{~nm}$, the smallest thickness for which superconduc- 


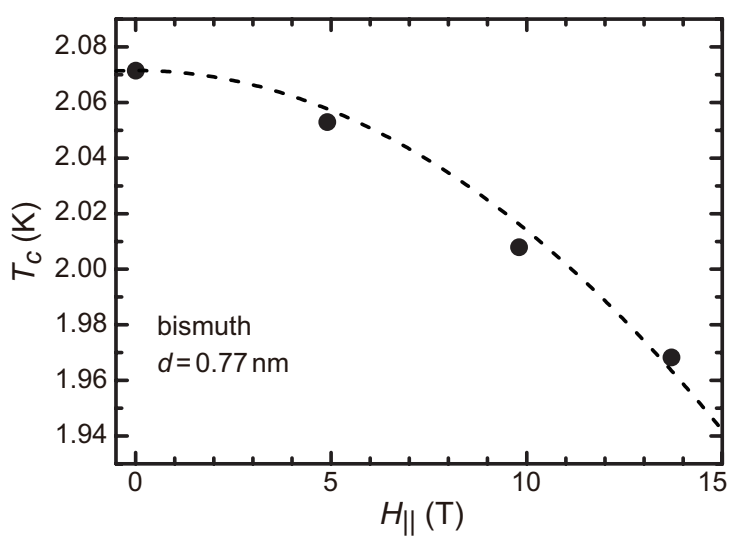

Fig. 5. $H_{\|}$dependence of $T_{c}$ for the Bi film with $d=0.77 \mathrm{~nm}$. The dashed line is the best quadratic fit.

tivity was observed. Since this value is a few times larger than the cube root of the volume per atom in bulk $\mathrm{Bi}(0.33 \mathrm{~nm})$, the film is not considered to be a monolayer. Thus, it is not appropriate to apply the theory developed for two-dimensional systems. On the other hand, the $H_{\|}$dependence of $T_{c}$ was found to be very weak. The suppression of $T_{c}$ is larger, but comparable to that in the $\mathrm{Pb}$ films. ${ }^{10)}$ The Rashba spin-orbit interaction may also play an essential role in the ultrathin film of Bi with $Z=83$, which is close to $Z=82$ for $\mathrm{Pb}$.

\subsection{Al films}

In the case of $\mathrm{Al}$ films, the smallest thickness for the observation of superconductivity was $0.82 \mathrm{~nm}$, which is larger than the cube root of the volume per atom in bulk $\mathrm{Al}(0.26 \mathrm{~nm})$. At $d=0.68 \mathrm{~nm}, R_{s q}$ exhibits an insulating temperature dependence as shown in the inset of Fig. 6. In order to discuss the morphology of the quench-condensed Al films, we study the magnetoresistance of the insulating film in the perpendicular direction. In Fig. 6, the data at $T=0.58 \mathrm{~K}$ are shown together with those for the $\mathrm{Pb}$ film in the submonolayer regime $(d=0.16 \mathrm{~nm})$. While the $H_{\perp}$ dependence of $R_{s q}$ is positive for the $\mathrm{Pb}$ film, it is negative for the $\mathrm{Al} \mathrm{film}$. Such strong negative magnetoresistance has been reported for granular $\mathrm{Pb}$ films on fire-polished glass or quartz substrates, where the electrical conduction is dominated by quasiparticle tunneling between superconducting grains. ${ }^{27)}$ The negative magnetoresistance in the insulating regime is explained as a result of the enhancement of the conductance due to the suppression of the superconducting energy gap by the magnetic field. It seems plausible to suppose that the negative magnetoresistance observed in the present $\mathrm{Al}$ film results from the same mechanism and that the morphology is granular, ${ }^{28)}$ while the monolayer $\mathrm{Pb}$ films are considered to be homogeneously disordered on GaAs(110). On the other hand, the negative magnetoresistance in the Al film survives even in high magnetic fields where the superconducting state is expected to be destroyed. Further study is required to understand the origin of the negative magnetoresistance in the insulating regime.

Figure 7 shows the $H_{\|}$dependence of $T_{c}$ for the Al film with $d=0.82 \mathrm{~nm}$. In contrast to the case of the Bi film shown in Fig. 5, $T_{c}$ decreases rapidly with increasing $H_{\|}$. Since the spin-orbit interaction is very small in $\mathrm{Al}(Z=13)$, the critical magnetic field is expected to be determined by the Pauli para-

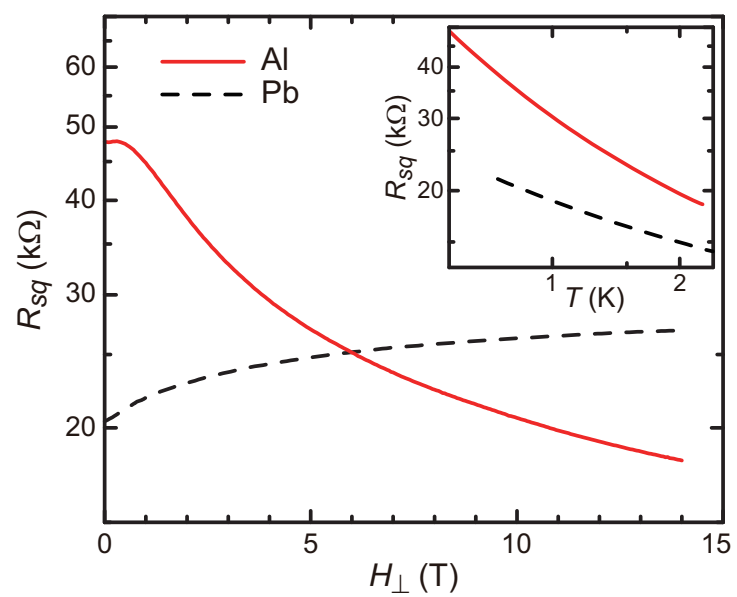

Fig. 6. (Color online) $H_{\perp}$ dependence of the sheet resistance. The solid (red) curve is obtained for the Al film with $d=0.68 \mathrm{~nm}$ at $T=0.58 \mathrm{~K}$. The dashed curve is obtained for the Pb film with $d=0.16 \mathrm{~nm}\left(n=5.4 \mathrm{~nm}^{-2}\right)$ at $T=0.83 \mathrm{~K}$. The inset shows the $T$ dependence of $R_{s q}$ at $H=0$.

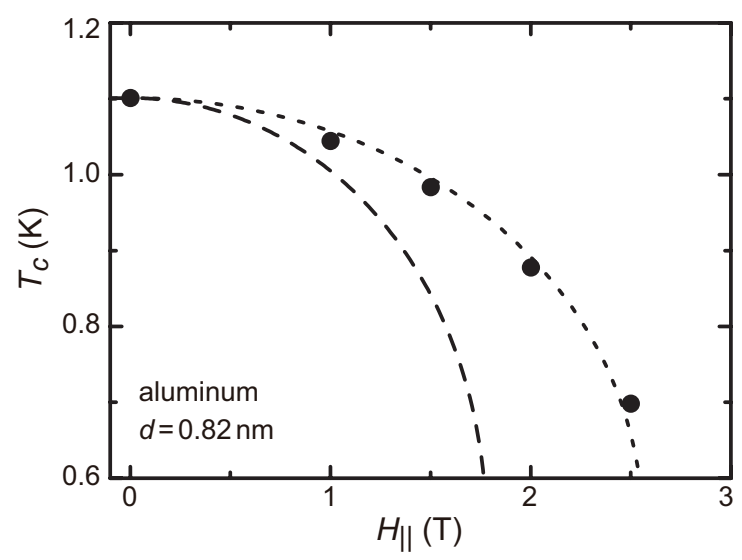

Fig. 7. $H_{\|}$dependence of $T_{c}$ for the Al film with $d=0.82 \mathrm{~nm}$. The dashed curve is the second-order phase transition lines calculated from Ref. 30. The dotted curve is obtained from the dashed curve by multiplying its abscissas by 1.44 .

magnetic limit. The phase transition due to the paramagnetic effect is of the second order for $T_{c} \geq 0.56 T_{c 0} .^{29,30)}$ The calculated phase boundary shown as the dashed curve in Fig. 7 qualitatively reproduces the experimental behavior. While the energy gap at $T=0$ is given by $\Delta_{0}=1.76 k_{B} T_{c 0}$ in the theory, it was shown in Ref. 31 that disorder can lead to an increase in the $\Delta_{0} / k_{B} T_{c 0}$ ratio. If this is the case, the phase boundary may shift toward a higher $H_{\|}$. The dotted curve was obtained by multiplying $H_{\|}$for the dashed curve by 1.44 so as to fit the experimental data.

\section{Summary}

As reported in this paper, we have studied the effect of a parallel magnetic field on the superconductivity of ultrathin metal films grown on a cleaved GaAs(110) surface. In monolayer In films, the $H_{\|}$dependence of $T_{c}$ is much stronger than that expected from Eq. (1), which was derived for $\Delta_{R} \gg$ $\hbar \tau^{-1}$. We extended the analysis of Refs. 8 and 9 to the case $\Delta_{R} \lesssim \hbar \tau^{-1}$. The theory well reproduces the experimental results when we use $\Delta_{R} \approx 0.04 \mathrm{eV}$, which is one order of mag- 
nitude smaller than that expected for monolayer $\mathrm{Pb}$ films. In a few-monolayer Bi film, the suppression of $T_{c}$ with increasing $H_{\|}$is very weak and comparable to that in the monolayer $\mathrm{Pb}$ films. In the $\mathrm{Al}$ film, on the other hand, $T_{c}$ decreases rapidly with increasing $H_{\|}$as expected from the simple calculation of the Pauli paramagnetic effect. By comparing the experimental results for different $Z$, it has been confirmed that a strong spin-orbit interaction is essential for the robustness of superconductivity against $H_{\|}$.

This work has been partially supported by Grants-in-Aid for Scientific Research (A) (No. 21244047) and (B) (No. 26287072) from MEXT, Japan.

1) T. Zhang, P. Cheng, W. J. Li, Y. J. Sun, G. Wang, X. G. Zhu, K. He, L. L. Wang, X. C. Ma, X. Chen, Y. Y. Wang, Y. Liu, H. Q. Lin, J. F. Jia, and Q. K. Xue, Nat. Phys. 6, 104 (2010).

2) T. Uchihashi, P. Mishra, M. Aono, and T. Nakayama, Phys. Rev. Lett. 107, 207001 (2011).

3) E. I. Rashba, Sov. Phys. Solid State 2, 1109 (1960).

4) Yu. A. Bychkov and E. I. Rashba, JETP Lett. 39, 78 (1984).

5) V. Barzykin and L. P. Gor'kov, Phys. Rev. Lett. 89, 227002 (2002).

6) P. Fulde and R. A. Ferrell, Phys. Rev. 135, A550 (1964).

7) A. I. Larkin and Yu. N. Ovchinnikov, Zh. Eksp. Teor. Fiz. 47, 1136 (1964) [Sov. Phys. JETP 20, 762 (1965)].

8) O. V. Dimitrova and M. V. Feigel'man, JETP Lett. 78, 637 (2003).

9) O. Dimitrova and M. V. Feigel'man, Phys. Rev. B 76, 014522 (2007).

10) T. Sekihara, R. Masutomi, and T. Okamoto, Phys. Rev. Lett. 111, 057005 (2013).

11) A. M. Clogston, Phys. Rev. Lett. 9, 266 (1962).

12) B. S. Chandrasekhar, Appl. Phys. Lett. 1, 7 (1962).

13) $\mathrm{The}_{\mathrm{RuO}}$ resistance thermometer (RO-600) was manufactured by Scientific Instruments, Inc., which offers a simple linear formula for a positive magnetoresistance. It allows the correction of any apparent temperature, measured in a magnetic field of up to $16 \mathrm{~T}$, to the actual temperature with an error of less than $\pm 1.6 \%$ for a broad temperature range of 0.036 to $4.2 \mathrm{~K}$. Our calibration agrees with the formula within the error bars.

14) D. B. Haviland, Y. Liu, and A. M. Goldman, Phys. Rev. Lett. 62, 2180 (1989).
15) The elastic scattering time was roughly estimated from $R_{N}=$ $\left(\alpha n_{e} e^{2} \tau / m_{e}\right)^{-1}$ in the same manner as used in Ref. 10. Here, $n_{e}$ is the free-electron value of the electron density equal to the valence electron density of indium $\left(n_{e}=3 n\right), m_{e}$ is the free-electron mass, and $\alpha=0.27$ is the correction factor.

16) In the presence of an asymmetric confining potential in the $z$ direction and a magnetic field in the $x$ direction, the Cooper pairs have a nonzero momentum $q$ in the $y$ direction so that the total free energy is reduced owing to the Rashba term. ${ }^{8,9}$ ) The nonzero $q$ leads to phase oscillations of the superconducting order parameter. On the other hand, $q$ and the free-energy reduction are very small, and we do not take them into account in the calculation of $T_{c}$.

17) K. Maki, Phys. Rev. 148, 362 (1966).

18) R. A. Klemm, A. Luther, and M. R. Beasley, Phys. Rev. B 12, 877 (1975).

19) M. I. D’yakonov and V. I. Perel, Zh. Eksp. Teor. Fiz. 60, 1954 (1971) [Sov. Phys. JETP 33, 1053 (1971)].

20) I. Žutić, J. Fabian, and S. Das Sarma, Rev. Mod. Phys. 76, 323 (2004).

21) Note that these values have some uncertainty due to the uncertainty in the estimation of $\tau$.

22) I. Gierz, T. Suzuki, E. Frantzeskakis, S. Pons, S. Ostanin, A. Ernst, J. Henk, M. Grioni, K. Kern, and C. R. Ast, Phys. Rev. Lett. 103, 046803 (2009).

23) S. Hatta, T. Aruga, Y. Ohtsubo, and H. Okuyama, Phys. Rev. B 80, 113309 (2009).

24) K. Sakamoto, H. Kakuta, K. Sugawara, K. Miyamoto, A. Kimura, T. Kuzumaki, N. Ueno, E. Annese, J. Fujii, A. Kodama, T. Shishidou, H. Namatame, M. Taniguchi, T. Sato, T. Takahashi, and T. Oguchi, Phys. Rev. Lett. 103, 156801 (2009).

25) K. Yaji, Y. Ohtsubo, S. Hatta, H. Okuyama, K. Miyamoto, T. Okuda, A. Kimura, H. Namatame, M. Taniguchi, and T. Aruga, Nat. Commun. 1, 17 (2010).

26) L. Petersen and P. Hedegård, Surf. Sci. 459, 49 (2000).

27) R. P. Barber, Jr., S. Y. Hsu, J. M. Valles, Jr., R. C. Dynes, and R. E. Glover, Phys. Rev. B 73, 134516 (2006).

28) Y. Liu, D. B. Haviland, B. Nease, and A. M. Goldman, Phys. Rev. B 47, 5931 (1993).

29) G. Sarma, J. Phys. Chem. Solids 24, 1029 (1963).

30) K. Maki and T. Tsuneto, Prog. Theor. Phys. 31, 945 (1964).

31) B. Sacépé, C. Chapelier, T. I. Baturina, V. M. Vinokur, M. R. Baklanov, and M. Sanquer, Phys. Rev. Lett. 101, 157006 (2008). 